\title{
THE EFFECT OF METFORMIN MONOTHERAPY ON BIOCHEMICAL PARAMETERS ASSOCIATED WITH DIABETES MELLITUS, KIDNEY, CARDIAC, LIVER, THYROID, AND REPRODUCTIVE ORGAN FUNCTIONS IN PRE- AND POST-MENOPAUSE WOMEN WITH TYPE 2 DIABETES MELLITUS
}

\author{
ARUN KUMAR D ${ }^{1}$, ARIVAZHAGAN R ${ }^{2}$, SUDHANDIRAN G ${ }^{3}$, SANGEETHA R ${ }^{1}$, SWAMINATHAN ${ }^{4 *}$ \\ ${ }^{1}$ Department of Biochemistry, Vels University, Pallavaram, Chennai - 600 117, Tamil Nadu, India. ${ }^{2}$ Department of Biochemistry, \\ Cancer Institute Adyar, Chennai - 600 020, Tamil Nadu, India. ${ }^{3}$ Department of Biochemistry, University of Madras, Guindy Campus, \\ Chennai - 600 025, Tamil Nadu, India. ${ }^{4}$ Department of Biochemistry, Apollo Speciality Hospitals, Vanagaram, Chennai - 600 095, Tamil \\ Nadu, India. Email: glorynathan@gmail.com
}

Received: 02 November 2017, Revised and Accepted: 25 November 2017

ABSTRACT

Objective: The aim of this study was to find out the alterations in biochemical parameters associated with the functioning of kidney, cardiac, liver, thyroid, and reproductive organs in pre- and post-menopause Type 2 diabetes mellitus (T2DM) women treated with metformin monotherapy (MMT) and to select a set of biochemical tests as routine to evaluate the functions of the above organs at regular intervals of time based on the levels of various biochemical parameters during a period of 1 year at 6 and 12 months.

Method: A total of 100 T2DM patients (50 pre- and 50 post-menopause women) who visited Apollo Speciality Hospitals Vanagaram, Sugar Clinic, and who were on treatment with MMT and 100 (50 pre- and 50 post-menopause age-matched non-diabetic women) were enrolled for this study. Fully automated analyzers and reagents and controls were used for all the assays to ensure the validity of the results obtained. GraphPad online calculator was used to calculate $t$ and $p$ values.

Results: MMT for menopause women with T2DM had improved the diabetic parameters, and the levels of fasting plasma glucose (FPG), postprandial plasma glucose (PPPG), and glycosylated hemoglobin (HbA1c) have dropped from $179.48 \mathrm{mg} / \mathrm{dL}, 270.8 \mathrm{mg} / \mathrm{dL}$, and 8.75\% at diagnosis to 135.36 , 199.45, and 7.05, respectively, after 12 months of MMT. In the case of post-menopause, the corresponding levels were 180.6, 263.2, and 8.85 to 133.79, 189.06 , and $6.68 \%$, respectively, and all the five organ functions were also not altered as the parameters tested for each organ function returned to normal. The drop in all the parameters levels was significant compared to the levels at diagnosis and the $\mathrm{p}$ values ranged from $<0.01$ to $<0.0001$.

Conclusion: Metformin treatment has shown very good improvement in the progressive reductions in the levels of FPG, PPPG, and HbA1c as well as maintaining the functions of kidney, cardiac, liver, thyroid, and reproductive organs to near normal as per the associations of these organ-specific parameters before and after 6 and 12 months of MMT.

Keywords: Diabetes mellitus, Metformin, Menopause, PG, Glycosylated hemoglobin, Chronic kidney disease, Cardiovascular disease, Thyroidstimulating hormone, Luteinizing hormone, Follicle stimulating hormone, Prolactin.

(C) 2018 The Authors. Published by Innovare Academic Sciences Pvt Ltd. This is an open access article under the CC BY license (http://creativecommons. org/licenses/by/4. 0/) DOI: http://dx.doi.org/10.22159/ajpcr.2018.v11i3.23447

\section{INTRODUCTION}

Diabetes mellitus (DM) is a growing epidemic in a developing country like India. According to the World Health Organization statistics, the numbers of people living with DM are increasing at the rate of $8 \%$ per year in India. Epidemiological survey indicates that Type $1 \mathrm{DM}$ (T2DM) is prevalent in both pre- and post-menopause women compared to Type 1 DM in the same group. Such women encounter many complications such as hyperglycemia, metabolic syndrome (MetS), oxidative stress (OS), obesity, hypertension (HT), and dyslipidemia. In spite of antidiabetic drug treatment many pre- and post-menopause women may encounter alteration in the functioning of kidney, cardiac, liver, thyroid, and reproductive organs and it depends on the drug used [1].

Many brands of antidiabetic drugs are being used to control T2DM including pre- and post-menopause women. The prevalence of T2DM is found to be higher in post-menopause women compared to premenopause. As T2DM is a complex and progressive disorder, there is general agreement for the use of metformin as the first line of treatment since many studies have proved its tolerability. It has been proved that the best initial pharmacological treatment of T2DM patients is MMT, and it was found to be effective in controlling T2DM as well as regulating the functioning of the organs listed above [2].
If metformin is not contraindicated, it will be the first line of treatment for T2DM. The selection of antidiabetic drugs is mainly based on tolerability, cost, side effects, hypoglycemic risk, comorbidities, and sometimes on patient preference. Some contraindications exist for some drugs on those with impaired renal, liver, and heart functions in the use of metformin and glitazones. Lacunae still exist in the use of antidiabetic drugs in patients with renal, cardiovascular disease (CVD), heart failure, and diabetic retinopathy. Hence, it is important to select the appropriate antidiabetic drug for patients who have comorbidities for the above disease states [3].

For T2DM, antidiabetic drug treatments are the method of choice. Many brands of hypoglycemic agents are available, but it is important to take into account, use and tolerability as well as proven efficacy in disease management. Among the various antidiabetic drugs used to treat T2DM, sitagliptin, exenatide and Exubera offer a new approach to assisting patients with T2DM [4].

In most Indian diabetic clinics, sulfonylurea and metformin are the drugs of choice to treat T2DM; however, their effect on lipid profile is poorly understood. Combined treatment with metformin plus glimepiride showed improvement in lipid status for Indian population with T2DM [5]. 
The most common risk factor for DM is chronic kidney disease (CKD), and treatments for such patients are very complicated. In the past, many efficacy studies on CKD have been excluded, and data on safety and efficacy are missing. Metformin is a globally accepted drug of choice to treat T2DM as this drug shows a low risk of hypoglycemia, modest weight loss together with its efficacy and low cost and less cardiovascular mortality. The use of sulfonylurea is to be avoided for patients with active hepatic metabolites as they are excreted through kidney and such patients are to be treated with dipeptidyl peptidase four inhibitors [6].

For T2DM patients with advanced CKD and dialysis, alpha-glucosidase inhibitors should be avoided as per National Kidney Foundation kidney disease outcome studies. In general, the oral antidiabetic treatments differ for different countries. Dipeptidyl peptidase four inhibitors maybe the drug of choice in future for patients with T2DM since this drug was found to be suitable for T2DM patients with CKD [7].

In T2DM patients, low high-density lipoprotein cholesterol (HDL-c) is prevalent and treatment with antidiabetic drug generally reduces glycosylated hemoglobin (HbA1c) level and increases HDL-c levels. Hence, glucose control in the form of reduced HDL-c was found to correlate with increased HDL-c, and hence, persistent HDL-c dysfunction in spite of decrease in HbA1c level may contribute to residual CVD risk in T2DM patients [8].

T2DM patients treated with metformin shows significant alteration of pro-inflammatory markers through a mediatory pathway by reducing the levels of C-reactive protein (CRP), and Interleukin levels there by risk factors associated with CVD and Stroke in T2DM patients [9].

Pre-diabetic is an intermediate stage between normal and clinical diabetes. It is important to start treating pre-diabetes otherwise it may lead to the development of CVD, pioglitazone, an insulin sensitizer, when used for T2DM patients, showed a protective effect on atherosclerosis development [10].

As early as in 1950, it has been proved that an inverse correlation exists between HDL-c and coronary heart disease (CHD) and this observation later led to therapeutic modification of HDL-c levels. Framingham and other studies have predicted that for every $10 \mathrm{mg} / \mathrm{dL}$ increase in HDL-c, $2-3 \%$ reduction in the risk of CVD was observed. Although statin therapy may reduce low-density lipoprotein cholesterol (LDL-c) in CVD patients with T2DM, still cardiovascular morbidity and mortality may occur. Hence, new strategies and therapeutic options are needed to reduce the risk for CVD, and many research findings in this aspect show controversial outcome. It may be due to HDL-c particle functionality and not on HDL-c levels that assess the effect on inflammation and hemostasis [11].

Uncontrolled DM, generally, increase the risk of CVD, but still, solid information on this is lacking with respect to the lipid profile levels. The significant independent predictors of CVD identified are age, renal albuminuria, and low HDL-c, and raised LDL-c which accounts to $12 \%$ risk for CVD. Even when total cholesterol (TC) is normal, increased LDL-c is found to be a strong predictor of CVD risk in patients with DM [12].

CVD has been found to be linked to an oxidized form of LDL-c and a role for myeloperoxidase oxidized (MOX-LDL) LDL-c dysfunction may be the cause for CVD risk. MOX-LDL may interfere with some parameters associated with angiogenesis leading to high LDL-c levels, and in some patients, it may impair the endothelial cell function which may contribute to negative progression of the atheroma plague [13].

In conventional diagnosis for ischemic heart disease (IHD), TC, TC/ HDL-c ratios are mostly used. In obese patients, LDL-c/HDL-c may underestimate IHD risk when triglycerides (TGs) levels are high. It was found that for a given LDL-c/HDL-c ratio, TC/HDL-c ratio was found to be higher for men in the hypertriglyceridemia than among men with normal TGs. Features of insulin resistance (IR), Apo lipoprotein-B, and LDL-c particle risk may be the reason behind for such observations noted above. Hence, it is recommended to make use of TC/HDL-c as well as LDL-c/HDL-c ratios to assess the alterations in metabolic indices predicting IHD, and it may be related to IR syndrome and LDL-c/HDL-c ratio [14]

In the diagnosis of subclinical hypothyroidism ( $\mathrm{SCH})$, lipid profile, especially LDL-c ratio, lipoprotein-a, and hs-CRP may be made use of in the evaluation while individual lipid profile parameters did not show any correlation between patients and controls, LDL-c/HDL-c ratio and hs-CRP showed high significance between the groups. No significant was found between LP(a) and IR. Hence, LDL-c/HDL-c ratio may be useful in the diagnosis of SCH [15].

It has been shown that coronary artery disease is associated with low HDL-c and increased TGs levels along with small dense LDL-c particles. Low HDL-c along with elevated TGs may be a risk factor for CVD [16].

Non-alcoholic fatty liver disease (NAFLD) is a common finding in children with obese related comorbidities in relation to IR. Among obese Korean children, IR, alanine transaminase (ALT), lipid profile, body mass index (BMI), and blood pressure all showed significant correlation and in boys higher ALT was found to be independently associated with IR [17].

It is a common findings that liver dysfunction may contribute to the development of T2DM. Aspartate transaminase (AST), ALT and gammaglutamyl transpeptidase $(\gamma$-GT) were associated with body fat. ALT is found to be an important enzyme which was found to be associated with obesity, hepatic IR, decrease in hepatic Insulin Sensitivity, and the development of T2DM: and hence elevated serum ALT is a marker of risk for T2DM, and it may be a potential source for the pathogenesis of T2DM [18].

In a study, elevated $\gamma$-GT was found to be the predictor of T2DM in adults, however, the role of $\gamma$-GT in IR among children is largely unknown. Serum $\gamma$-GT and ALT but not AST were found to be positively associated with IR in boys and girls, and ALT emerged as the independent determinant of IR among Korean Children [19].

Both family history and elevated liver enzymes are found to be an independent marker of T2DM. Multiple regression analysis showed age, family history, FPG, and TGs were associated with $\gamma$-GT independent of the other variables. Increase in liver enzymes may play a role in increasing the risk for development of T2DM [20].

Recent studies have shown that $\gamma$-GT may play a pivotal role as a marker for alcohol-induced liver disease and it is said to play a role in the development of CVD through oxidative process leading to atherosclerosis. Further, some studies show that $\gamma$-GT is associated with MetS, IR, hyperlipidemia, and obesity, and the association to IR was found to be strong. It may be due to high prevalence of NAFLD in the population studied. Since the measurement of serum $\gamma$-GT is a lowcost test, it may be frequently used to find out association with some important risk factors of T2DM and CVD. Such association has been frequently found out between IR and NAFLD [21].

All prediabetic patients may have association to IR, and it may affect thyroid function, its size and the nodules. Thyroid-stimulating hormone (TSH) showed statistically significant difference between placebotreated group and metformin-treated group. There was no change in thyroid value in metformin-treated group compared to placebo group. Hence, in prediabetic group, metformin therapy decreases TSH only in people with TSH levels ${ }^{3} 2.5 \mathrm{mIU} / \mathrm{mL}$ and prevents the increase in thyroid values [22].

Many studies have established that TSH shows suppressive action of metformin in subjects in the overt or subclinical thyroid dysfunction, 
but it was not the case in normal individuals. Metformin may exhibit antimitogenic properties against thyroid cancer type molecules. However, experimental evidence shows reduced efficacy of radioactive treatment followed by metformin therapy, and it may limit the use of radioactive iodine therapy in thyroid cancer [23]

Metformin therapy for 12 weeks in patients with SCH showed a decrease in percent body fat compared to pre-treatment levels. Both insulin and IR decreased significantly, and a positive correlation between TSH and insulin as well as IR was observed. Hence, metformin suppresses serum TSH levels without affecting free thyroxin (FT4) and free triiodo threonine (FT3) levels in SCH group who do not have autoimmune thyroiditis [24].

In post-menopause women, follicular stimulating hormone (FSH) and luteinizing hormone ( $\mathrm{LH}$ ) levels showed association to estrogen, and such pattern was not observed in pre-menopause women. Both FSH and LH levels were found to be high in pre-menopause and is characteristic of pre-menopause status, and it precedes the sustained loss of sex hormone secretion by the aging ovary. However, post-menopause biochemical changes are no guarantee of post-menopause status [25].

Human menopause is directly linked to ovarian failure, and oocyte depletion and central nervous system may play a part. Women with no surge in FSH and LH experience more symptoms of menopause than women in whom estrogens increases are indicating failure of estrogen surges. In anovulatory cycle, follicular phase did not show LH secretion indicating decreased estrogen-negative feedback. Hence, hypothalamicpituitary insensitivity to estrogen in aging may be the reason for such observations in per-menopause [26].

Evidence linking gonadotropin to ovarian carcinogenesis is lacking. Higher level of FSH is said to decrease ovarian cancer risk compared to low FSH levels. Reduction in invasive epithelial ovarian cancer was observed among women with increased levels of circulating FSH. Hence, FSH measurement in menopause women may serve as a risk predictor for ovarian carcinogenesis [27].

A study has assessed the significance of LH/FSH ratio in pre and postmenopause women. An elevated LH/FSH ratio was frequently observed in polycystic ovarian syndrome (PCOS) women. Both obesity and IR are prevalent in PCOS. No solid evidence is still available on the usefulness of LH/FSH ratio outside the context of PCOS. In PCOS women, CRP was positively associated to $\mathrm{LH} / \mathrm{FSH}$ ratio in hyperglycemic patients it was $>2.0$, and in case of HDL-c $<50 \mathrm{mg} / \mathrm{dL}$, it was $>1.0$ and 2.0. Hence, in post-menopause women with markers for inflammations, dyslipidemia and PCOS associated morbidities, LH/FSH ratio is an important marker, but further studies are required to prove this [28]

Weight gain observed in the post-menopause women may be due to estrogen deficiency-induced increased appetite. In overweight women, a positive correlation was observed between FSH and BMI. Melatonin deficiency is attributed for this observation, and hence it is recommended to treat such patients with melatonin [29].

A study indicates that mean serum prolactin (PRL) decreased significantly in post-menopause women. A combined estrogen/ androgen, as well as calcitonin therapy in two group of post-menopause women, was found to influence PRL levels during a 2 years treatment while FSH and LH levels remained unchanged [30].

A study indicates that females, mostly post-menopause women who are found to be hyperprolactinemic may be normalized when treated with dopamine agonist therapy. $45 \%$ of post-menopause women underwent such therapy have normalized their PRL levels compared to $18 \%$ and $7 \%$ of pregnant and male patients treated with such dopamine agonist. Hence, all menopause women with hyperprolactinemia to be treated with dopamine agonist [31].

\section{METHODS}

This study is an open, parallel, prospective, non-randomized, concurrent control group, (experimental group before and after treatment) in comparison with sex and age-matched controls.

\section{Conforming to ethics}

Approval from Institutional Ethics Committee (Apollo Hospitals Chennai) was obtained for this study before the commencement of patient's recruitment. All the controls and patients data were identified by a unique identification number and patient initials. Patient's information and the results obtained on blood sample were kept confidential.

\section{Inclusion criteria}

- All female patients in the age group of 18-60 years.

- Who are newly diagnosed with T2DM during the past 3-6 months.

- The plasma glucose levels are FPG - >120 mg/dL and diagnosed as having T2DM based on clinical and laboratory diagnosis.

- $\mathrm{HbA} 1 \mathrm{c}$ is $>6.0 \%$

- The liver enzymes AST, ALT, alkaline phosphatase (ALP), and $\gamma$-GT are within normal levels

- Who are taking metformin oral hypoglycemic drug as monotherapy.

- Who do not have diseases associated with kidney, cardiac, liver, thyroid, and reproductive organs.

\section{Exclusion criteria}

- Females below the age of 18 years and above 60 years.

- Who have history of T2DM

- Pregnant women

- The plasma glucose levels are FPG - <120 mg/dL and PPPG - $<160 \mathrm{mg} / \mathrm{dL}$

- HbA1c value are $<6.0 \%$

- Who have diseases associated with kidney, cardiac, liver, thyroid, and reproductive organs.

- Patients are taking drugs for any other disease.

\section{Subject recruitment and data collection}

For the evaluation of biochemical parameters involved in this study, the research diabetic group was compared with normal group and/ individuals. Individuals for the research group were recruited from Apollo Speciality Hospitals referral from consultant sugar clinic. The normal group comprised willing individuals who were not diabetic. Both groups underwent blood tests, and the results were compared.

\section{Experimental group}

Newly diagnosed pre- and post-menopause women with T2DM in the age group of 18-60 years.

- Pre-menopause women: 50

- Post-menopause women: 50

\section{Control group}

Data were collected from age-matched pre and post-menopause nondiabetic normal women undergoing master health checkup.

- Pre-menopause women: 50

- Post-menopause women: 50

\section{Measurement of biochemical parameters}

For the measurement of Diabetic, Kidney, Liver, Cardiac, profiles, DIRUI CS 1300B fully automatic analyzer and kits supplied by Dialab were used. For thyroid (FT3, FT4, and TSH) and reproductive profiles (LH, FSH, and PRL) Siemens ADVIA Centaur CP Immunoassay Analyzer and kits supplied by the same company were used. For measuring HbA1c, Biorad D-10 (high-performance liquid chromatography method) instrument and kit supplied by them was used.

Biorad accuracy controls at two levels were used every time when assays were carried out to validate the accuracy of the results obtained in this study. 


\section{Statistical calculations}

A software www.graphpad.comdownloaded from the website was used to calculate $t$ and $p$ values.

\section{RESULTS}

The results obtained were subjected to statistical analysis using an online calculator downloaded from www.graphpad.com to get $\mathrm{t}$ and $\mathrm{p}$ values between groups for each parameter and the improvement after 6 and 12 months of MMT was assessed.

Table 1 summarize results obtained for patients at diagnosis are higher than the levels of normal controls for the diabetic profile, TC, LDL-c, and TGs (cardiac profile). Total protein, ALT, and $\gamma$-GT (liver profile), FT4 and TSH (thyroid profile), and FSH and PRL (reproductive profile). The levels of significance ranged from $<0.0001$ to $<0.01$.

Table 2 summarizes highly significant decrease in diabetic profile ( $\mathrm{p}<0.0001)$, cardiac profile ( $\mathrm{p} 0.0001$ to $<0.01)$, and significant decrease in liver profile, improvement in FT3, and FT4, as well as LH and FSH, were observed after 12 months of treatment compared to the levels at diagnosis.

Table 3 summarize except for creatinine, TGs, ALP, and LH/FSH ratio, all other parameters results were significantly different for patients compared to controls.

Table 4 summarize except for TSH and FSH all other parameters have shown significant/highly significant improvement/no alterations in the functioning of all five organs after 12 months of MMT.

\section{DISCUSSIONS}

The primary objective of this study was to evaluate the effect of MMT on pre- and post-menopausal women with T2DM, as assessed by clinical examination, BMI and waist-hip-ratio (WHR) at diagnosis, and 6 and 12 months after MMT. Although as many as 6 hypoglycemic drugs are available for use, the hospital where this study was undertaken uses mostly MMT for the majority of patients with T2DM including for pre- and post-menopausal women [3-5].

\section{Diabetic profile}

The first criteria undertaken in this study is to prove the clinical findings of the enrolled pre and post-menopause T2DM patients with the results obtained for FPG, PPPG, and HbA1c. All these three values were found to be higher for both pre- and post-menopause women with T2DM compared to the respective control groups [1].

\section{Urea and creatinine}

The results obtained for the diabetic people have shown improvement after 12 min of treatment. In this study, MMT was initiated as soon as the patients were diagnosed to have T2DM. The two selected kidney function parameters urea and creatinine values were close to the normal controls. Previous studies have shown that DKD is prevalent in uncontrolled DM of both types due to hyperglycemic and reduced metabolic alterations. However, we did not find any DKD as all the urea values obtained for 50 patients in both groups of T2DM did not show any increase in values. Studies have shown that MMT is contraindicated for CKD, but due to global acceptable of MMT as the drug of choice, our study did not predict any CKD in our population. Hence, in the T2DM populations studied some significant reduction in creatinine levels were observed in both groups of patients. Microvascular complications, as well as DN, observed in established CKD patients with T2D were not observed in our population $[6,7]$.

\section{Lipid profile}

Among the lipid profile, TC showed good improvement and a drop of around $40 \mathrm{mg} / \mathrm{dL}$ in both pre- and post-menopause women after 12 months of treatment, showing a significant level of $<0.0001$. However, previous studies did not mention about the changes in TC after MMT, but they mentioned the importance of TC in CVD. 50\% of patients with TC levels $<250 \mathrm{mg} / \mathrm{dL}$ still may develop CHD. A highly significant drop in TC was observed for post-menopause after 12 months of MMT. Earlier studies have pointed out that persistent HDL-c dysfunction despite improved HDL-c can contribute to residual CVD in T2DM patients $[3,10]$.

Table 1: Pre-menopause control group versus pre-menopause at diagnosis

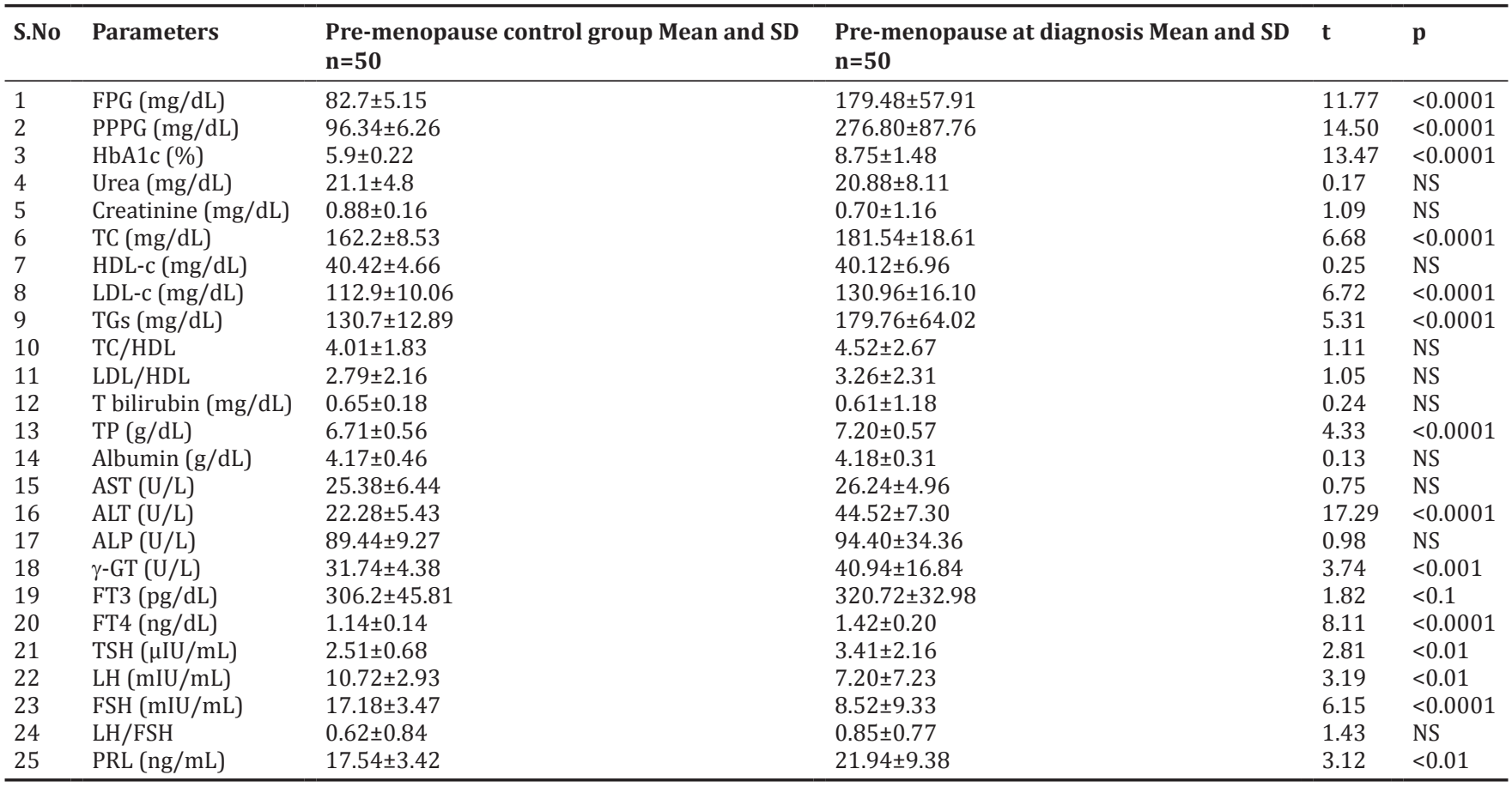

FPG: Fating plasma glucose, PPPG: Postprandial plasma glucose, HbA1c: Glycosylated hemoglobin, TC: Total cholesterol, HDL-c: High-density lipoprotein cholesterol, LDL-c: Low-density lipoprotein cholesterol, TGs: Triglycerides, TP: Total protein, AST: Aspartate transaminase, ALT: Alanine transaminase, ALP: Alkaline phosphatase, $\gamma$-GT: Gamma glutamyl transpeptidase, FT3: Free tri iodo threonine, FT4: Free thyroxin, TSH: Thyroid-stimulating hormone, LH: Leutinizing hormone, FSH: Follicular stimulating hormone, PRL: Prolactin, SD: Standard deviation, NS: Non significant 
Table 2: Pre-menopause at diagnosis versus pre-menopause after 12 months treatment

\begin{tabular}{|c|c|c|c|c|c|}
\hline S.No & Parameters & $\begin{array}{l}\text { Pre-menopause at diagnosis Mean and SD } \\
n=50\end{array}$ & $\begin{array}{l}\text { Pre-menopause after } 12 \text { months Mean and SD } \\
n=40\end{array}$ & $\mathbf{t}$ & $\mathbf{p}$ \\
\hline 1 & $\mathrm{FPG}(\mathrm{mg} / \mathrm{dL})$ & $179.48 \pm 57.91$ & $135.36 \pm 41.94$ & 4.04 & 0.0001 \\
\hline 2 & PPPG (mg/dL) & $276.80 \pm 87.76$ & $199.45 \pm 58.17$ & 4.79 & $<0.0001$ \\
\hline 3 & $\operatorname{HbA1c}(\%)$ & $8.75 \pm 1.48$ & $7.05 \pm 1.13$ & 5.99 & $<0.0001$ \\
\hline 4 & Urea $(\mathrm{mg} / \mathrm{dL})$ & $20.88 \pm 8.11$ & $19.3 \pm 5.39$ & 1.06 & NS \\
\hline 5 & Creatinine $(\mathrm{mg} / \mathrm{dL})$ & $0.70 \pm 1.16$ & $0.59 \pm 0.11$ & 0.60 & NS \\
\hline 6 & $\mathrm{TC}(\mathrm{mg} / \mathrm{dL})$ & $181.54 \pm 18.61$ & $140.68 \pm 15.73$ & 11.07 & $<0.0001$ \\
\hline 8 & LDL-c (mg/dL) & $130.96 \pm 16.10$ & $97.75 \pm 19.31$ & 8.90 & $<0.0001$ \\
\hline 9 & TGs (mg/dL) & $179.76 \pm 64.02$ & $113.58 \pm 35.87$ & 5.79 & $<0.0001$ \\
\hline 10 & $\mathrm{TC} / \mathrm{HDL}$ & $4.52 \pm 2.67$ & $3.29 \pm 0.46$ & 2.88 & $<0.01$ \\
\hline 11 & LDL/HDL & $3.26 \pm 2.31$ & $2.23 \pm 0.44$ & 2.78 & $<0.01$ \\
\hline 12 & T bilirubin (mg/dL) & $0.61 \pm 1.18$ & $0.55 \pm 0.12$ & 0.32 & NS \\
\hline 13 & $\mathrm{TP}(\mathrm{g} / \mathrm{dL})$ & $7.20 \pm 0.57$ & $6.04 \pm 0.37$ & 11.13 & $<0.0001$ \\
\hline 14 & Albumin (g/dL) & $4.18 \pm 0.31$ & $3.49 \pm 0.25$ & 11.41 & $<0.0001$ \\
\hline 15 & $\operatorname{AST}(\mathrm{U} / \mathrm{L})$ & $26.24 \pm 4.96$ & $24.63 \pm 4.80$ & 1.55 & NS \\
\hline 18 & $\gamma-\mathrm{GT}(\mathrm{U} / \mathrm{L})$ & $40.94 \pm 16.84$ & $27.56 \pm 7.78$ & 4.64 & $<0.0001$ \\
\hline 19 & FT3 (pg/dL) & $320.72 \pm 32.98$ & $263.84 \pm 25.73$ & 8.95 & $<0.0001$ \\
\hline 20 & FT4 (ng/dL) & $1.42 \pm 0.20$ & $1.18 \pm 0.17$ & 6.04 & $<0.0001$ \\
\hline 21 & TSH $(\mu \mathrm{IU} / \mathrm{mL})$ & $3.41 \pm 2.16$ & $3.42 \pm 2.17$ & 0.02 & NS \\
\hline 22 & $\mathrm{LH}(\mathrm{mIU} / \mathrm{mL})$ & $7.20 \pm 7.23$ & $14.67 \pm 5.49$ & 5.40 & $<0.0001$ \\
\hline 23 & FSH (mIU/mL) & $8.52 \pm 9.33$ & $17.06 \pm 8.47$ & 4.49 & $<0.0001$ \\
\hline 24 & $\mathrm{LH} / \mathrm{FSH}$ & $0.85 \pm 0.77$ & $0.82 \pm 0.42$ & 0.22 & NS \\
\hline 25 & PRL (ng/mL) & $21.94 \pm 9.38$ & $8.66 \pm 6.15$ & 7.72 & $<0.0001$ \\
\hline
\end{tabular}

FPG: Fating plasma glucose, PPPG: Postprandial plasma glucose, HbA1c: Glycosylated hemoglobin, TC: Total cholesterol, HDL-c: High-density lipoprotein cholesterol, LDL-c: Low-density lipoprotein cholesterol, TGs: Triglycerides, TP: Total protein, AST: Aspartate transaminase, ALT: Alanine transaminase, ALP: Alkaline phosphatase, $\gamma$-GT: Gamma-glutamyl transpeptidase, FT3: Free triiodo threonine, FT4: Free thyroxin, TSH: Thyroid-stimulating hormone, LH: Leutinizing hormone, FSH: Follicular stimulating hormone, PRL: Prolactin, SD: Standard deviation, NS: Non significant

Table 3: Post-menopause control group versus post-menopause at diagnosis

\begin{tabular}{|c|c|c|c|c|c|}
\hline S.No & Parameters & $\begin{array}{l}\text { Post-menopause control group Mean and SD } \\
n=50\end{array}$ & $\begin{array}{l}\text { Post-menopause at diagnosis Mean and SD } \\
n=50\end{array}$ & $\mathbf{t}$ & $\mathbf{p}$ \\
\hline 1 & $\mathrm{FPG}(\mathrm{mg} / \mathrm{dL})$ & $92.8 \pm 6.95$ & $180.6 \pm 62.01$ & 9.95 & $<0.0001$ \\
\hline 2 & PPPG (mg/dL) & $96.48 \pm 4.94$ & $263.2 \pm 84.22$ & 13.97 & $<0.0001$ \\
\hline 3 & HbA1c $(\%)$ & $5.92 \pm 0.22$ & $8.85 \pm 1.58$ & 12.98 & $<0.0001$ \\
\hline 4 & Urea $(\mathrm{mg} / \mathrm{dL})$ & $27.04 \pm 4.64$ & $22.82 \pm 8.57$ & 3.06 & $<0.01$ \\
\hline 5 & Creatinine $(\mathrm{mg} / \mathrm{dL})$ & $0.95 \pm 0.14$ & $1.05 \pm 0.36$ & 1.83 & $<0.10$ \\
\hline 6 & $\mathrm{TC}(\mathrm{mg} / \mathrm{dL})$ & $162.7 \pm 6.43$ & $181 \pm 39.89$ & 3.20 & $<0.01$ \\
\hline 8 & LDL-c (mg/dL) & $112.2 \pm 13.89$ & $136.6 \pm 18.56$ & 7.44 & $<0.0001$ \\
\hline 9 & TGs (mg/dL) & $163.7 \pm 6.84$ & $169.8 \pm 74.02$ & 0.58 & NS \\
\hline 10 & TC/HDL & $3.85 \pm 1.40$ & $4.82 \pm 1.40$ & 3.46 & $<0.001$ \\
\hline 11 & LDL/HDL & $2.66 \pm 3.02$ & $3.65 \pm 0.85$ & 2.23 & $<0.05$ \\
\hline 12 & $\mathrm{~T}$ bilirubin $(\mathrm{mg} / \mathrm{dL})$ & $0.81 \pm 0.16$ & $0.56 \pm 0.10$ & 9.34 & $<0.0001$ \\
\hline 13 & $\mathrm{TP}(\mathrm{g} / \mathrm{dL})$ & $7.03 \pm 0.39$ & $7.2 \pm 0.46$ & 1.99 & $<0.05$ \\
\hline 14 & Albumin (g/dL) & $3.68 \pm 0.40$ & $4.14 \pm 0.17$ & 7.48 & $<0.0001$ \\
\hline 15 & AST (U/L) & $25.76 \pm 4.50$ & $29.5 \pm 6.31$ & 3.41 & $<0.001$ \\
\hline 17 & ALP (U/L) & $88.66 \pm 6.87$ & $92.64 \pm 23.75$ & 1.13 & NS \\
\hline 18 & $\gamma-\mathrm{GT}(\mathrm{U} / \mathrm{L})$ & $26.98 \pm 5.32$ & $41.78 \pm 10.87$ & 8.64 & $<0.0001$ \\
\hline 19 & FT3 (pg/dL) & $295.8 \pm 49.0$ & $251.5 \pm 72.88$ & 3.56 & $<0.001$ \\
\hline 20 & FT4 (ng/dL) & $1.18 \pm 0.28$ & $1.62 \pm 0.36$ & 6.82 & $<0.0001$ \\
\hline 21 & $\mathrm{TSH}(\mu \mathrm{IU} / \mathrm{mL})$ & $4.55 \pm 0.51$ & $3.32 \pm 3.67$ & 2.34 & $<0.05$ \\
\hline 22 & $\mathrm{LH}(\mathrm{mIU} / \mathrm{mL})$ & $35.8 \pm 8.37$ & $13.02 \pm 9.00$ & 13.10 & $<0.0001$ \\
\hline 23 & $\mathrm{FSH}(\mathrm{mIU} / \mathrm{mL})$ & $65.42 \pm 10.43$ & $44.14 \pm 27.09$ & 5.18 & $<0.0001$ \\
\hline 24 & $\mathrm{LH} / \mathrm{FSH}$ & $0.55 \pm 0.80$ & $0.36 \pm 0.18$ & 1.63 & NS \\
\hline 25 & PRL (ng/mL) & $13.48 \pm 3.08$ & $18.01 \pm 11.12$ & 2.77 & $<0.01$ \\
\hline
\end{tabular}

FPG: Fating plasma glucose, PPPG: Postprandial plasma glucose, HbA1c: Glycosylated hemoglobin, TC: Total cholesterol, HDL-c: High-density lipoprotein cholesterol, LDL-c: Low-density lipoprotein cholesterol, TGs: Triglycerides, TP: Total protein, AST: Aspartate transaminase, ALT: Alanine transaminase, ALP: Alkaline phosphatase, $\gamma$-GT: Gamma-glutamyl transpeptidase, FT3: Free triiodo threonine, FT4: Free thyroxin, TSH: Thyroid-stimulating hormone, LH: Leutinizing hormone, FSH: Follicular stimulating hormone, PRL: Prolactin, SD: Standard deviation, NS: Non significant

A reduction in HDL-c as pointed out in earlier studies may be possible only if IR, HT, and MetS were present in the patients under treatment and all these factors were minimal in our population, and hence MMT has normalized the levels of both TC and HDL-c. Many studies have predicted that HDL-c is the chief parameter to assess CVD and development and it was proved in Framingham's study. Regarding LDL-c, the levels were at the upper limit of normal for pre- and post-menopause T2DM patients during diagnosis and before starting therapy $[8,11]$. 
Table 4: Post-menopause at diagnosis versus post-menopause after 12 months treatment

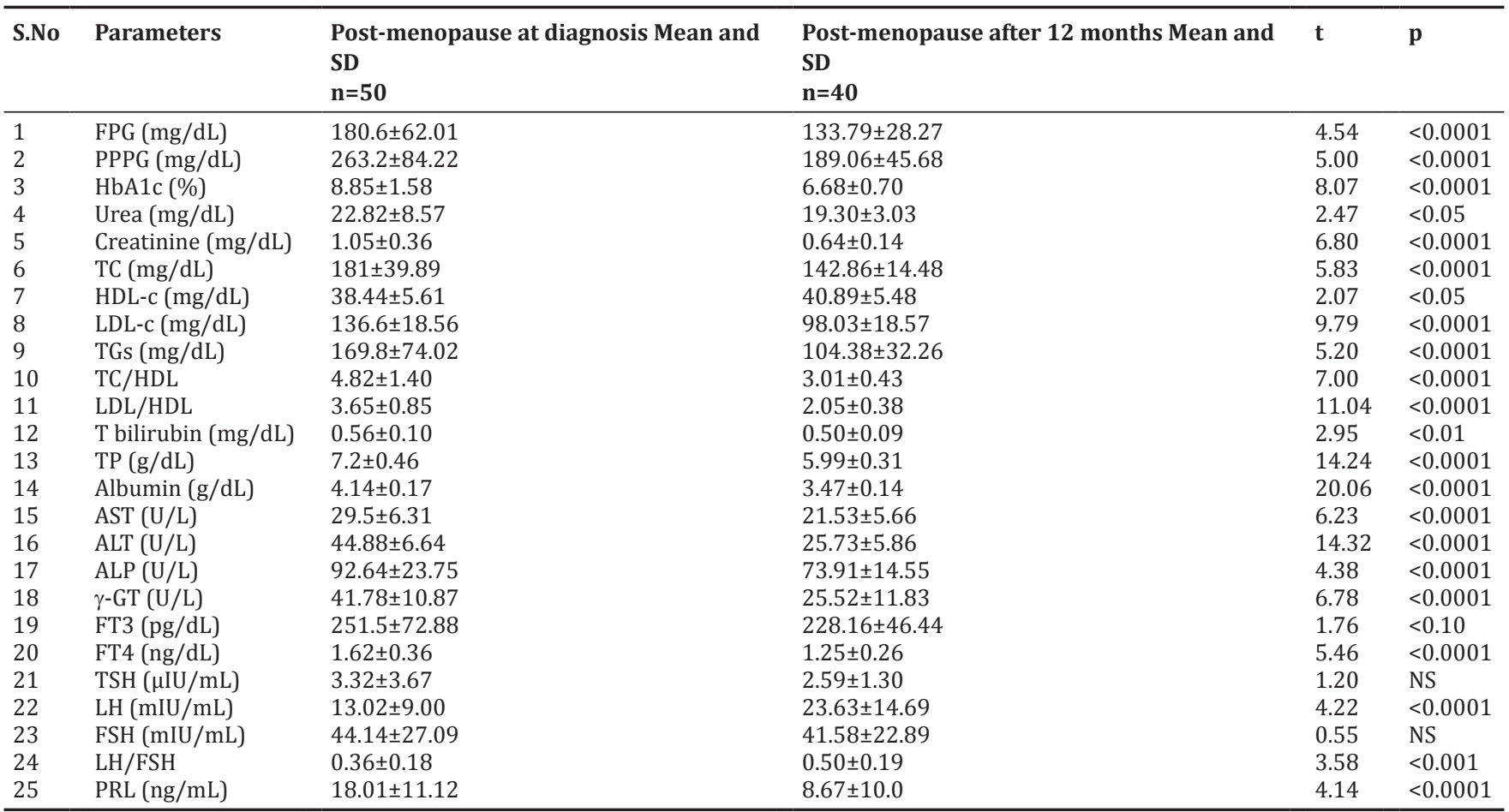

FPG: Fating plasma glucose, PPPG: Postprandial plasma glucose, HbA1c: Glycosylated hemoglobin, TC: Total cholesterol, HDL-c: High-density lipoprotein cholesterol, LDL-c: Low-density lipoprotein cholesterol, TGs: Triglycerides, TP: Total protein, AST: Aspartate transaminase, ALT: Alanine transaminase, ALP: Alkaline phosphatase, $\gamma$-GT: Gamma-glutamyl transpeptidase, FT3: Free triiodo threonine, FT4: Free thyroxin, TSH: Thyroid-stimulating hormone, LH: Leutinizing hormone, FSH: Follicular stimulating hormone, PRL: Prolactin, SD: Standard deviation, NS: Non significant

Current guideline recommends aggressive strategic treatment to reduce LDL-c, but in our study simple use of MMT, which is a good medication has improved LDL-c reducing all complications associated with cardiac functions. Among HDL-c and LDL-c, the former should increase, and the later decrease and every $10 \mathrm{mg} / \mathrm{dL}$ increase in LDL-c are associated with $12 \%$ increase in CVD $[12,13]$.

The two calculated parameters, TC/HDL-c and LDL/HDL-c, did not have enough mention in earlier studies; however, we included these two ratios to compare with the prevalent normal values for these ratios. Both groups of patients did not show any symptoms of CVD and were never referred to cardiologists. This study outcome is unable to explain this unusual findings but future studies are showing extended time after 12 months could possible establish the reasons and, may be the ratios will improve after 12 months of treatment. Previous studies have shown a correlation between LDL/HDL ratio to thyroid hormones, but we did not undertake this comparison [13-15].

Highly significant reductions in TGs were observed for both pre- and post-menopause women at diagnosis and after 12 months treatment. MMT, indeed showed lowering effect of TGs, a substance which may contribute to atherogenic index of plasma, which if not controlled may lead to CVD. Some studies predicted that the association between TGs and CVD may improve if HDL-c improves. Hence, TGs may serve as a predictive parameter for CVD. The outcome of our study are inconsistent with previous studies [11,14 16].

\section{Liver function tests}

To evaluate the alterations/improvement in LFT, a total of 7 important tests were done in all groups of patients. Pre-menopause women with T2DM at diagnosis and after treatment with MMT, did not encounter any type of jaundice, possibly due to no alterations in liver functions. Among the liver enzymes studied, while AST levels remained more or less close to normal values. ALT, which is more specific than AST as a liver-specific enzyme showed a drop in value after 12 months of treatment for pre- menopause during the same period after MMT. Studies have shown that ALT as the best index for the evaluation of LFT in T2DM and elevation in ALT is a risk for T2DM as it was found to be associated to BMI, WHR, and lipid profile. Both AST and ALT serves as the best-combined liver enzymes for predicting progress/improvement in T2DM, and both the enzymes showed associations to T2DM. Elevation in these two enzymes, if not controlled will lead to NAFLD $[17,18]$.

Values for $\gamma$-GT were at the upper limit of normal for post-menopause patients. After treatment with MMT, the values dropped for the above two groups of patients. Previous studies showed that during screening for T2DM, $\gamma$-GT served as the predictor for the development of T2DM; however, the role of $\gamma$-GT in IR has not yet been established. Among the elevations in liver enzymes in T2DM, $\gamma$-GT prevails over other enzymes, and it is more sensitive in predicting the presence of T2DM. Further, $\gamma$-GT serves as a marker in suspected cases of NAFLD along with obesity. Our study findings in the levels of $\gamma$-GT were in agreement with these observations and it tally with previous studies [19-21].

Although ALP is also grouped among liver enzymes, its level mostly is used to distinguish the three types of jaundices. However, after MMT, significant drop in ALP levels have also been observed, for postmenopause at diagnosis and after12 months therapy for both groups. Previous studies have shown reductions and are inconsistent with the earlier observations [20].

\section{Thyroid hormones}

FT3 levels were at the median limit of normal for both pre- and post-menopause women. However, previous studies have shown no changes in both FT3 and FT4 after 12 weeks of MMT, but our findings showed drop in values after 12 months. In our study too, we found out some decrease in FT3 after 6 months compared to diagnosis, but the decrease in values are not statistically significant $[15,22]$. FT4, one of the important thyroid hormones which are usually done along with $\mathrm{TSH}$, all values were found to be perfectly normal, but at the lower 
level. The value for FT4 dropped for both groups of patients. However, this observations were not inconsistent with previous findings. Many studies conducted in the past have reported TSH lowering effect by MMT, but our study did not find such effects. It may be due to the fact that the patients selected by us had already normal TSH and hence no further decrease in TSH levels was observed after 12 months of MMT $[23,24]$

\section{Reproductive hormones}

Previous studies have shown an increase in LH and FSH values in premenopausal women with T2DM, and the outcome of our research is found to be inconsistent with such observations. In post-menopausal women, decrease in LH and FSH were observed due to aging ovaries and some studies have linked BMI to both LH and FSH, but we did not find such findings in our study. The only prove that this study had found out improvement in the levels of LH due to MMT and more research are required on this aspect in future. With respect to FSH levels, we observed that the pre-treatment levels for pre- and post-menopause at diagnosis and after 12 months treatment showed a significant difference for pre and NS for post-menopausal women. These findings were found to be inconsistent with earlier studies as FSH levels in postmenopause are subjected to variations due to menstrual history and aging $[25,26]$.

Post-menopause LH/FSH ratio showed improvement after 12 months therapy compared to pre-treatment levels. In previous studies, it was reported that increased glucose levels increase the ratio to $>2.0$ and were found to be inversely related to LDL-c, but we did not work out for such correlations since glucose levels showed improvement after 12 months of MMT. Since treatment with MMT had shown improvement in glucose and HbA1c levels, the ratio was $<1.0[27,28]$.

Hyper prolactinemia is a common finding in all reproductive disorders, especially in infertility. In both cases, a decrease in values was observed. In terms of statistical significance, the p values observed were $<0.0001$ for both groups suggesting that MMT has improved hyperprolactinemia observed in both groups. It is very difficult to predict the improvement in absolute value, and our findings have proved improvement in PRL levels using $p$ values which were found to be very good at a level of $<0.0001$. All these above observations have been predicted in many earlier studies $[30,31]$.

\section{CONCLUSIONS}

This study has established that MMT is a safe and effective treatment for menopause women with T2DM. FPG, PPPG, and HbA1c levels at diagnosis have decreased from $179.48 \mathrm{mg} / \mathrm{dL}, 276.8 \mathrm{mg} / \mathrm{dL}$ and $8.75 \%$ to $135.36 \mathrm{mg} / \mathrm{dL}, 199.45 \mathrm{mg} / \mathrm{dL}$, and $7.05 \%$ for pre-menopause and the corresponding levels were $180.6,263.2$ and $8.85 \%$ to 133.79 , 189.06 , and $6.68 \%$ for post-menopause, respectively. The significant drop observed in the above levels showed a $\mathrm{p}<0.0001$ for all groups. The parameters associated with the five organs function also showed decrease in values at the end of 12 months therapy and the $p$ values for these parameters at diagnosis, and at the end of 12 months, MMT ranged from $<0.01$ to $<0.0001$. This study therefore strongly recommends that MMT is a safe and effective treatment for pre- and post-menopause women with T2DM.

\section{ACKNOWLEDGMENT}

The authors would like to thank the management of Apollo Speciality Hospitals, especially Dr. Mitra Ghosh, Chief of Lab Services, Apollo Speciality Hospitals at Vanagaram, for giving permission to carry out this work.

\section{REFERENCES}

1. Sjoberg-Tuominen L, Tiitinen A. Menopause in a diabetic. Duodecim 2009;125:2689-94

2. Tschöpe D, Hanefeld M, Meier JJ, Gitt AK, Halle M, Bramlage P, et al. The role of co-morbidity in the selection of antidiabetic pharmacotherapy in Type-2 diabetes. Cardiovasc Diabetol 2013;12:62.
3. Fung CS, Wan EY, Wong CK, Jiao F, Chan AK. Effect of metformin monotherapy on cardiovascular diseases and mortality: A retrospective cohort study on Chinese Type 2 diabetes mellitus patients. Cardiovasc Diabetol 2015;14:137.

4. Ibrahim R. Diabetes mellitus Type II: Review of oral treatment options. Int J Pharm Pharmsci 2010;2 Suppl 1:21-30.

5. Ingle PV, Talele GS. Comparative effects of metformin in combination with glimepiride and glibenclamide on lipid profile in Indian patients with Type 2 diabetes mellitus. Int J Pharm Pharm Sci 2011;3 Suppl 5:472-4

6. Ioannidis I. Diabetes treatment in patients with renal disease: Is the landscape clear enough? World J Diabetes 2014;5:651-8.

7. Abe M, Okada K, Soma M. Antidiabetic agents in patients with chronic kidney disease and end-stage renal disease on dialysis: Metabolism and clinical practice. Curr Drug Metab 2011;12:57-69.

8. Fadini GP, Iori E, Marescotti MC, de Kreutzenberg SV, Avogaro A. Insulin-induced glucose control improves HDL cholesterol levels but not reverse cholesterol transport in Type 2 diabetic patients. Atherosclerosis 2014;235:415-7.

9. Khalaf BH, Abdulridha MK, Kadhim KA, Hussain SR. Influence of larged aily doses of metformin on the expression of interleukin-1B and $\mathrm{C}$-reactive protein in Type 2 diabetes patients. Int J Pharm Pharm Sci 2014:6:506-9.

10. Verma RK, Tripathi P, Pandey AK. Role of pioglitazone on progression of atherosclerosis in prediabetes: A mini review. Int J Pharm Pharm Sci 2014;6:13-5.

11. Mahdy Ali K, Wonnerth A, Huber K, Wojta J. Cardiovascular disease risk reduction by raising HDL cholesterol - current therapies and future opportunities. Br J Pharmacol 2012;167:1177-94.

12. Howard BV, Robbins DC, Sievers ML, Lee ET, Rhoades D, Devereux RB, et al. LDL cholesterol as a strong predictor of coronary heart disease in diabetic individuals with insulin resistance and low LDL: The strong heart study. Arterioscler Thromb Vasc Biol 2000;20:830-5.

13. Daher J, Martin M, Rousseau A, Nuyens V, Fayyad-Kazan H, Van Antwerpen $\mathrm{P}$, et al. Myeloperoxidase oxidized LDL interferes with endothelial cell motility through miR-22 and hemeoxygenase induction: Possible involvement in reendothelialization of vascular injuries mediators inflamm 2014;2014:134635.

14. Lemieux I, Lamarche B, Couillard C, Pascot A, Cantin B, Bergeron J, et al. Total cholesterol/HDL cholesterol ratio vs LDL cholesterol/HDL cholesterol ratio as indices of ischemic heart disease risk in men: The quebec cardiovascular study. Arch Intern Med 2001;161:2685-92.

15. Mahto M, Chakraborthy B, Gowda SH, Kaur H, Vishnoi G, Lali P. Are hsCRP levels and LDL/HDL ratio better and early markers to unmask onset of dyslipidemia and inflammation in asymptomatic subclinical hypothyroidism. Indian J Clin Biochem 2012;27:284-9.

16. Halle M, Berg A, Baumstark MW, König D, Huonker M, Keul Joseph, et al. Influence of mild to moderately elevated triglycerides on low density lipoprotein subfraction concentration and composition in healthy men with low high density lipoprotein cholesterol levels. Atherosclerosis 1999;143:185-92.

17. Yoo J, Lee S, Kim K, Yoo S, Sung E, Yim J, et al. Relationship between insulin resistance and serum alanine aminotransferase as a surrogate of NAFLD (nonalcoholic fatty liver disease) in obese Korean children. Diabetes Res Clin Pract 2008;81:321-6.

18. Vozarova B, Stefan N, Lindsay RS, Saremi A, Pratley RE, Bogardus C, et al. High alanine aminotransferase is associated with decreased hepatic insulin sensitivity and predicts the development of Type 2 diabetes. Diabetes 2002;51:1889-95.

19. Lee SY, Sung E, Chang Y. Elevated serum gamma-glutamyltransferase is a strong marker of insulin resistance in obese children. Int $\mathrm{J}$ Endocrinol 2013;2013:578693

20. Inoue K, Matsumoto M, Miyoshi Y, Kobayashi Y. Elevated liver enzymes in women with a family history of diabetes. Diabetes Res Clin Pract 2008:79:e4-7.

21. Martins MC, Faleiro LL, Afonso B, Fonseca A. Association of gamma glutamyltransferase, metabolic syndrome and cardiovascular risk. Acta Med Port 2010;23:579-88.

22. Karimifar M, Aminorroaya A, Amini M, Mirfendereski T, Iraj B, Feizi A, et al. Effect of metformin on thyroid stimulating hormone and thyroid volume in patients with prediabetes: A randomized placebocontrolled clinical trial. J Res Med Sci 2014;19:1019-26.

23. Pappa T, Alevizaki M. Metformin and thyroid: An update. Eur Thyroid J 2013;2:22-8.

24. Rajput R, Saini M, Rajput M, Shankar V. Effects of metformin on thyroid function in patients of subclinical hypothyroidism. J Endocrinol 
Metab 2013:3:105-10.

25. Metcalf MG, Donald RA, Livesey JH. Pituitary-ovarian function in normal women during the menopausal transition. Clin Endocrinol (Oxf) 1981;14:245-55

26. Weiss G, Skurnick JH, Goldsmith LT, Santoro NF, Park SJ. Menopause and hypothalamic-pituitary sensitivity to Estrogen. JAMA 2004;292:2991-6.

27. McSorley MA, Alberg AJ, Allen DS, Allen NE, Brinton LA, Dorgan JE, et al. Pre diagnostic circulating follicle stimulating hormone (FSH) concentrations and ovarian cancer risk. Maturitas 2012;71:55-61.

28. Beydoun H, Beydoun M, Wiggins N, Stadtmauer L. Relationship of Obesity-related disturbances with $\mathrm{LH} / \mathrm{FSH}$ ratio among postmenopausal women with United states. Maturitas 2012;71:55-61.

29. Kapia EW, Chojnacki J, Stepien A, Wachowska-Kelly P, Klupinska G, Chojnacki C. Melatonin and female - Hormone secretion in postmenopausal women. Int J Mol Sci 2015;16:1030-42.

30. Balint-Peric LA, Prelevic GM. Changesin prolactin leels with the menopause; The effect of Estrogen/Androgen and calcitonin treatment. Gynecol Endocrinol 1997;11:275-80.

31. Karunakaran S, Page RC, Wass JA. The effect of the menopause on prolactin levels in patients with hyperprolactinaemia. Clin Endocrinol (Oxf) 2001;54:295-300. 